\title{
Mutation screening in patients with syndromic craniosynostoses indicates that a limited number of recurrent FGFR2 mutations accounts for severe forms of Pfeiffer syndrome
}

\author{
Elisabeth Lajeunie ${ }^{1}$, Solange Heuertz ${ }^{1}$, Vincent El Ghouzzi ${ }^{1}$, Jelena Martinovic ${ }^{1}$, \\ Dominique Renier ${ }^{2}$, Martine Le Merrer ${ }^{1}$ and Jacky Bonaventure*,1 \\ ${ }^{1}$ INSERM U 393, Hôpital Necker-Enfants malades, Paris, France; ${ }^{2}$ Département de Neurochirurgie, \\ Hôpital Necker-Enfants malades, Paris, France
}

Crouzon Syndrome (CS), Pfeiffer syndrome (PS) and the phenotypically related Jackson-Weiss (JW) variant are three craniosynostotic conditions caused by heterozygous mutations in Fibroblast Growth Factor Receptor (FGFR) genes. Screening a large cohort of 84 patients with clinical features of CS, PS or JW by direct sequencing of genomic DNA, enabled FGFR1, 2 or 3 mutation detection in 79 cases. Mutations preferentially occurred in exons 8 and 10 of FGFR2 encoding the third Ig loop of the receptor. Among the 74 FGFR2 mutations that we identified, four were novel including three missense substitutions causing CS and a $\mathbf{2}$ bp deletion creating a premature stop codon and producing JW phenotype. Five FGFR2 mutations were found in one of the two tyrosine kinase subdomains and one in the Ig I loop. Interestingly, two FGFR2 mutations creating cysteine residues (W290C and Y340C) caused severe forms of PS while conversion of the same residues into another amino-acid (W290G/R, Y340H) resulted in Crouzon phenotype exclusively. Our data provide conclusive evidence that the mutational spectrum of FGFR2 mutations in CS and PS is wider than originally thought. Genotype-phenotype analyses based on our cohort and previous studies further indicate that in spite of some overlap, PS and CS are preferentially accounted for by two distinct sets of FGFR2 mutations. A limited number of recurrent amino-acid changes (W290C, Y340C, C342R and S351C) is commonly associated with the most severe Pfeiffer phenotypes of poor prognosis.

European Journal of Human Genetics (2006) 14, 289-298. doi:10.1038/sj.ejhg.5201558; published online 18 January 2006

Keywords: FGFR1; FGFR2; FGFR3; Pfeiffer syndrome; Crouzon syndrome; mutations

\section{Introduction}

Crouzon (MIM\# 123500), Jackson-Weiss (JW) (MIM\# 123150) and Pfeiffer syndromes (PS) (MIM\# 101600) are three clinically related craniosynostoses with common

*Correspondence: Dr J Bonaventure, INSERM U393, Hôpital Necker, 149 rue de Sèvres, 75743 Paris cedex 15, France.

Tel: + 331444940 00; Fax: + 331473485 14;

E-mail: bonavent@necker.fr

This study is dedicated to the memory of Elisabeth Lajeunie-Renier Received 28 April 2005; revised 4 October 2005; accepted 5 October 2005; published online 18 January 2006 clinical features including craniosynostosis, ocular hypertelorism with proptosis and midface hypoplasia. They differ by the absence in Crouzon syndrome (CS) and presence in PS and JW of limb abnormalities. Until recently, clinical distinction between PS and JW was based on the presence in JW of broad great toes with medial deviation and tarsal-metatarsal coalescence in the absence of hand anomalies. However, owing to clinical overlap between the two entities, JW syndrome is now considered as a clinical variant in the novel Nosology of Constitutional Disorders of Bone. ${ }^{1}$ PS has been subdivided into 
three clinical types. ${ }^{2}$ Type I, the most frequent form is usually associated with a benign course and satisfactory prognosis, whereas type II and III represent severe forms of the disease commonly associated with a poor outcome leading to early demise in some cases. Subdivision was based on the presence (type II) or absence (type III) of cloverleaf skull.

In 1994, CS has been ascribed to de novo mutations in the Fibroblast Growth Factor Receptor (FGFR) 2 gene. ${ }^{3}$ FGFR2 belongs to a family of four receptors comprising an extracellular ligand-binding domain, a transmembrane domain and an intracellular domain carrying the tyrosine kinase activity. Further studies have shown that FGFR2 mutations also accounted for Apert syndrome (AS, MIM\# 101200), JW, Beare Stevenson cutis gyrata syndrome (MIM\# 123790) and PS, ${ }^{4-8}$ but genetic heterogeneity of this latter syndrome was demonstrated by the identification in several families of a recurrent FGFR1 mutation causing mild forms of the disease. ${ }^{9,10}$ Sporadic cases of AS, CS and PS have been associated with advanced paternal age and the origin of FGFR2 mutations in these three conditions was demonstrated to be exclusively paternal. ${ }^{11,12}$

Surprisingly, the same FGFR2 mutation can give rise to CS, PS or JW phenotype. ${ }^{6,13}$ Until 2002, FGFR2 mutations had been identified almost exclusively in the extracellular domain of the receptor mainly in exons 8 (IIIa) and 10 (IIIc) encoding the third immunoglobulin-like (Ig III) loop and appeared to account for only $50 \%$ of CS and PS cases. ${ }^{14}$ In 2002, novel mutations in other regions of the receptor including the IgII loop and the tyrosine kinase subdomains TK1 and TK2 have been reported. ${ }^{15}$ Likewise, this study demonstrated that in a clinically homogeneous group of CS and PS patients, FGFR2 mutations were detectable in more than $90 \%$ of all cases, thus making the existence of an additional major locus very unlikely. ${ }^{14}$ A recurrent mutation, A391E, in a third gene, FGFR3, accounts for a peculiar form of CS associating craniosynostosis with acanthosis nigricans also called crouzonodermoskeletal syndrome. ${ }^{16}$

Molecular screening in a large cohort of AS, CS, JW variants and PS patients diagnosed in our hospital was performed. The diagnosis was based on the clinical presentation of the proband and family members. Mutations in one of the three FGFR genes (FGFR 1, 2 or 3) were found in 126/131 (96\%) of our cases. Four novel FGFR2 mutations were identified. FGFR2 mutations, although clustered mainly in exons 8 and 10, were also present with a lower frequency in exons 3,14 and 16. The recurrent FGFR3 mutation A391E in exon 10 caused CS with acanthosis nigricans whereas the P252R FGFR1 mutation produced PS with slight facial anomalies and absence of neurological defects. Altogether our results confirm that AS, CS and JW variants are genetically homogeneous at the FGFR2 locus whereas PS is heterogeneous, being caused by FGFR1 or FGFR2 mutations. Genotype-phenotype analysis based on our cohort and previous reports indicate that CS is caused by a wide spectrum of amino-acid substitutions in
FGFR2, some of which being specific. PS is preferentially accounted for by a different set of FGFR2 mutations. Clinical and radiological examination of PS patients further revealed that FGFR2 mutations creating cysteine residues at positions 290, 340 and 351 are associated with the most severe phenotypes.

\section{Materials and methods Patients and samples}

During the past 10 years, clinical diagnosis and surgical correction of the skull deformation in patients with syndromic craniosynostoses has been achieved by the same team of physicians and surgeons at the Craniofacial Surgery Department of the Hôpital Necker-Enfants Malades allowing us to obtain relevant clinical and radiological data and to collect blood samples for DNA analysis. Few cases were made available through the Foetopathological Unit of our hospital after ultrasound detection of severe craniosynostotic conditions and therapeutic termination of pregnancies. Each patient showed characteristic clinical features including synostosis of one or several cranial sutures, ocular proptosis, maxillary hypoplasia and midface retrusion. Clinical and radiological diagnosis was based on the following features: All patients with AS exhibited high forehead, ocular proptosis and syndactyly of the four limbs. Most Crouzon patients had brachycephaly, proptosis and moderate faciostenosis but were distinguishable from PS and JW variants by the absence of hand and foot anomalies. Pfeiffer patients had marked proptosis and faciostenosis. Their thumbs and halluces were broad and deviated with variable degree of soft tissue syndactyly (Table 3 ). Among 2594 children with proven craniosynostosis, 116 children from 100 families were diagnosed as Crouzon patients and 35 children from 33 families as Pfeiffer patients. Diagnosis of JW variants was established in two patients based on the presence of tarsal-metatarsal coalitions, broad and deviated halluces but normal hands. In our series, the birth prevalence of AS was $1 / 60000$ and CS was $1 / 50000$, this latter entity accounting for $4.5 \%$ of all craniosynostoses. PS was less frequent with an estimated birth prevalence of $1 / 150000$ and accounted for $1.3 \%$ of all craniosynostoses. DNA samples were obtained from 131 patients diagnosed as Apert, Crouzon, PSs or JW variants. Informed consents for molecular studies and photographs were obtained from all patients or their parents.

\section{Mutation analysis}

Molecular analysis was performed on a cohort of patients comprising 47 sporadic cases of Apert syndrome, 62 unrelated CS (20 familial cases and 42 sporadic cases) including two patients with acanthosis nigricans (one sporadic and one familial case), two familial cases of JW variants and 20 unrelated PS (two familial cases and 18 sporadic cases). 
PCR amplification of genomic DNA was performed using previously described primers and conditions. ${ }^{5,15}$ PCR fragments were directly sequenced on an ABI 3100 capillary sequencer (Applied Biosystems) with BigDye terminator mix. Since FGFR2 mutations preferentially occur in exons 8 and 10, these two exons were tested first. Novel mutations were validated by sequencing exons 8 and 10 in unaffected parents and 65 control genomic DNAs. When no mutation was found, screening of other exons was undertaken. Exon numbering was based on a recently proposed nomenclature. ${ }^{17}$ GenBank accession numbers for FGFR1: BC015035; FGFR2: AF410480; FGFR3: AY768549.

\section{RT-PCR amplifications}

For RT-PCR studies, total RNA was extracted from cultured human skin fibroblasts and control keratinocytes using the RNeasy Mini Kit (Qiagen). Complementary DNA was synthesized by priming with either random hexamers or oligo-dT in the presence of MuLV reverse transcriptase using the manufacturer's protocol (GeneAmp RNA PCR Core Kit, Roche). In all, 25-35 PCR cycles were then applied to amplify fragments of 281 and 286 bp specific for either the IIIb isoform (exon 9) or the IIIc isoform (exon 10) of FGFR2. For this purpose a common primer 5'-CACAGTGGTCGGAGGAGA-3' (F) located in exon 8 (IIIa) was used with either primer $5^{\prime}$-GTTTTGGCAGGACAGT GAGC-3' (R) to amplify the IIIb isoform or 5'-AGTTA CATTCCGAATATAGAG-3' (R) for the IIIc isoform. Sense and antisense primers used for GAPDH amplification were as follows: 5'-CATGTGGGCCATGAGGTCCACCAC- $3^{\prime}$ and 5'-CATGTGGGCCATGAGGTCCACCAC-3'. Samples were analysed on $1 \%$ agarose gels. Specificity of all RT-PCR products was tested by direct sequencing.

\section{Results}

Mutation screening was performed by direct sequencing of FGFR1, 2 and 3 genes. In FGFR1 and FGFR3 genes, sequence analyses were restricted to exons 7 and 10 respectively, whereas 16 exons of the FGFR2 gene were studied. FGFR mutations were detected in 126/131 (96\%) unrelated patients (Table 1). The recurrent FGFR1 mutation P252R was recorded in three Pfeiffer cases (one familial and two sporadic). In two Crouzon patients with acanthosis nigricans, the recurrent A391E FGFR3 mutation was identified. All other mutations causing AS, CS, JW variant and PS were found in the FGFR2 gene. As expected, mutations in exons 8 and 10 of the FGFR2 gene were largely predominant in our cohort, representing 95\% $(115 / 121)$ of the overall FGFR2 mutations. Among the 68 mutations in the Ig IIIa/Ig IIIc region causing CS or PS, 35 (51\%) either created or eliminated cysteine residues, thus generating unpaired cysteines putatively able to induce disulfide bond formation between two mutant receptors. Mutations outside exons 8 and 10 (6/74) were located in either exon 3 encoding the first Ig-like loop (Y105C) or exons 14 and 16 encoding the tyrosine kinase subdomains. Mutations N549H in the TK1 and K659N in the TK2 subdomains are homologous, respectively, to the N540K and K650N FGFR3 mutations causing hypochondroplasia. In five patients (three CS, one JW variant and one PS) no FGFR2 mutation was found. Analysis of exon 7 of FGFR1, exons 7 and 10 of FGFR3 and exon 1 of TWIST 1 also failed to reveal an abnormal sequence in those cases.

\section{Apert patients}

Our series comprised 47 patients with AS, of which 36 have been previously reported ${ }^{18}$ and 11 cases including three foetuses, were collected during the last 5 years. The S252W mutation was present in 29 patients (62\%). In all, 17 carried the P253R substitution (36\%) and exhibited severe phenotypes characterized by syndactyly of the five digits and mental retardation. The double nucleotide substitution converting serine 252 into phenylalanine (S252F) occurred in one case $(2 \%)$.

Table 1 Patients analyzed for FGFR mutations

\begin{tabular}{|c|c|c|c|c|c|}
\hline Clinical diagnosis & Number of patients & $\begin{array}{c}\text { Patients with FGFR2 } \\
\text { mutation }\end{array}$ & $\begin{array}{c}\text { Patients with FGFR1 } \\
\text { mutation }\end{array}$ & $\begin{array}{c}\text { Patients with FGFR3 } \\
\text { mutation }\end{array}$ & $\begin{array}{l}\text { Patients with no } \\
\text { FGFR mutation }\end{array}$ \\
\hline Apert syndrome & $47^{a}$ & $47(100 \%)$ & 0 & 0 & 0 \\
\hline Crouzon syndrome & $60^{b}$ & $57(95 \%)$ & 0 & 0 & $3(5 \%)$ \\
\hline Pfeiffer syndrome & $20^{c}$ & $16(80 \%)$ & $3(15 \%)$ & 0 & $1(5 \%)$ \\
\hline $\begin{array}{l}\text { Crouzon syndrome } \\
\text { with } \mathrm{AN}^{\mathrm{d}}\end{array}$ & 2 & 0 & 0 & 2 & 0 \\
\hline $\begin{array}{l}\text { Jackson-Weiss } \\
\text { phenotype }\end{array}$ & 2 & 1 & 0 & 0 & 1 \\
\hline Total & 131 & $121(92 \%)$ & $3(2.3 \%)$ & $2(1.5 \%)$ & $5(3.8 \%)$ \\
\hline
\end{tabular}

${ }^{a}$ Among our series of 47 patients with Apert syndrome, 36 have been previously reported. ${ }^{18}$

${ }^{\mathrm{b}}$ Five patients with CS previously reported by Ma et al ${ }^{19}$ were included in this series.

${ }^{\mathrm{C}}$ Two patients with PS previously reported by Lajeunie et al ${ }^{5}$ were included in this series.

${ }^{\mathrm{d}} \mathrm{AN}=$ acanthosis nigricans. 


\section{Crouzon patients}

Missense FGFR2 substitutions were detected in 57/60 unrelated cases including 17 familial forms and 40 sporadic cases. Five familial cases have been reported previously. ${ }^{19}$ In familial forms, presence of the single base change was confirmed by DNA sequencing of at least one additional affected member.

The Y105C mutation in exon 3 was found in one familial case. Three other family members carried the same substitution. In all, 19 missense mutations corresponding to 11 distinct heterozygous amino-acid changes were identified in exon 8. Two of these mutations (I288N and Y308C) are novel (Table 2). They were found neither in the DNA of unaffected parents, nor in 65 control DNA samples.
A total of 34 mutations corresponding to 11 distinct amino-acid changes occurred in exon 10. The most frequent mutation $\mathrm{C} 342 \mathrm{Y}$ was detected in 12 unrelated cases $(22 \%)$

Clinical re-examination of Crouzon patients carrying FGFR2 mutations showed that proptosis although variable was systematically present in all patients. Coronal sutures were the most frequently affected as $86 \%$ of patients presented bicoronal fusions (brachycephaly), 18\% exhibited both coronal and sagittal fusions (oxycephaly) and 5\% showed pansynostosis (fusion of all sutures). In the remaining 9\%, fusion was restricted to the sagittal (scaphocephaly) or the sagittal and lambdoid sutures. No correlation was found between the mutation and the

Table 2 FGFR mutations identified in the study

\begin{tabular}{|c|c|c|c|c|c|c|c|c|}
\hline Gene & Nucleotide change & Mutation & Protein domain & Familial/sporadic & Crouzon & Pfeiffer & Crouzon $+A N^{\mathrm{a}}$ & Jackson-Weiss \\
\hline FGFR2 & $314 A>G$ & Y105C & $\left.\lg \right|^{b}$ & $\mathrm{~F}$ & 1 & - & - & - \\
\hline FGFR2 & 799T $>C$ & S267P & $\lg$ IIIa & $S$ & 2 & - & - & - \\
\hline FGFR2 & $800 \mathrm{C}>\mathrm{T}$ & S267F & lg IIIa & $\mathrm{F}$ & 1 & - & - & - \\
\hline FGFR2 & $826 \mathrm{~T}>\mathrm{G}$ & F276V & lg IIIa & S & 1 & - & - & - \\
\hline FGFR2 & $833 G>T$ & $\mathrm{C} 278 \mathrm{~F}$ & lg IIIa & $S$ & 5 & - & - & - \\
\hline FGFR2 & $833 G>A$ & C278Y & lg IIla & $S$ & 1 & - & - & - \\
\hline FGFR2 & $863 \mathrm{~T}>\mathrm{A}$ & $1288 \mathrm{~N}$ & $\lg$ IIIa & $S$ & 1 & - & - & - \\
\hline FGFR2 & $866 \mathrm{~A}>C$ & Q289P & lg IIIa & $2 F+1 S$ & 3 & - & - & - \\
\hline FGFR2 & $868 \mathrm{~T}>\mathrm{G}$ & W290G & lg IIla & $\mathrm{F}$ & 1 & - & - & - \\
\hline FGFR2 & $868 G>C$ & W290R & lq IIIa & $S$ & 3 & & - & - \\
\hline FGFR2 & $870 \mathrm{G}>\mathrm{T}$ & W290C & lg IIIa & $S$ & - & 3 & - & - \\
\hline FGFR2 & $923 A>G$ & Y308C & $\lg$ IIIa & $S$ & 1 & - & - & - \\
\hline FGFR2 & $\begin{array}{l}940-2 \\
A>G\end{array}$ & Splice acceptor & $\lg$ IIIC & $S$ & - & 2 & - & - \\
\hline FGFR2 & $\begin{array}{l}\text { 958-959 } \\
\text { del AC }\end{array}$ & Premature stop codon & $\lg$ IIIC & $\mathrm{F}$ & - & - & - & 1 \\
\hline FGFR2 & $962 A>C$ & D321A & $\lg$ IIIC & $\mathrm{F}$ & - & 1 & - & - \\
\hline FGFR2 & $1009 G>C$ & A337P & $\lg$ IIIC & $S$ & 1 & - & - & - \\
\hline FGFR2 & $1012 G>C$ & G338R & lg IIIC & $3 \mathrm{~S}+1 \mathrm{~F}$ & 4 & - & - & - \\
\hline FGFR2 & $1018 \mathrm{~T}>\mathrm{C}$ & $\mathrm{Y} 340 \mathrm{H}$ & lg IIIC & $2 \mathrm{~S}+1 \mathrm{~F}$ & 3 & - & - & - \\
\hline FGFR2 & $1019 A>C$ & Y340S & $\lg$ IIIC & $S$ & 1 & - & - & - \\
\hline FGFR2 & $1019 A>C$ & Y340C & $\lg$ IIIC & $S$ & - & 2 & - & - \\
\hline FGFR2 & $1021 A>C$ & T341P & $\lg$ IIIC & $S$ & 1 & - & - & - \\
\hline FGFR2 & $1024 \mathrm{~T}>\mathrm{C}$ & C342R & lg IIIC & S & - & 1 & - & - \\
\hline FGFR2 & $1025 G>A$ & C342Y & lg IIIC & $9 S+3 F$ & 12 & - & - & - \\
\hline FGFR2 & $\begin{array}{l}1025 \mathrm{G}>\mathrm{C} / \\
1024 \mathrm{~T}>\mathrm{A}\end{array}$ & C342S & $\lg$ IIIC & $4 \mathrm{~S}+1 \mathrm{~F}$ & $1(\mathrm{~F})$ & $\begin{array}{l}3(S) \\
1(S)\end{array}$ & - & - \\
\hline FGFR2 & $1026 C>G$ & C342W & $\lg$ IIIC & $2 \mathrm{~F}+1 \mathrm{~S}$ & $2(\mathrm{~F})$ & $1(S)$ & - & - \\
\hline FGFR2 & $1032 \mathrm{G}>\mathrm{A}$ & A344A Cryptic site & lq IIIC & $3 F+2 S$ & 5 & - & - & - \\
\hline FGFR2 & $1040 C>C$ & S347C & $\lg$ IIIC & $\mathrm{S}$ & 2 & - & - & - \\
\hline FGFR2 & $1061 C>T$ & S354F & $\lg$ IIIC & $\mathrm{S}$ & 1 & - & - & - \\
\hline FGFR2 & $1070 \mathrm{~T}>\mathrm{C}$ & L357S & lg IIIC & $\mathrm{F}$ & 1 & - & - & - \\
\hline FGFR2 & $1645 A>C$ & $\mathrm{~N} 549 \mathrm{H}$ & TKK $^{\mathrm{c}}$ & $\mathrm{S}$ & 2 & 1 & - & - \\
\hline FGFR2 & $1922 A>C$ & K641R & TK2 & $\mathrm{S}$ & - & 1 & - & - \\
\hline FGFR2 & $1977 G>T$ & K659N & TK2 & $S$ & 1 & - & - & - \\
\hline FGFR1 & $755 C>G$ & P252R & Igll-IgIII linker & $2 \mathrm{~S}+1 \mathrm{~F}$ & - & 3 & - & - \\
\hline FGFR3 & $1172 C>A$ & A391E & $\mathrm{TM}^{\mathrm{d}^{3}}$ & $1 \mathrm{~S}+1 \mathrm{~F}$ & - & - & 2 & - \\
\hline
\end{tabular}

Nucleotide and amino acid numbers refer to the following GenBank accession numbers: FGFR1: BC 015035; FGFR2: AF 410480; FGFR3: AY 768549. Novel mutations are in bold.

${ }^{\mathrm{a}} \mathrm{AN}$ : acanthosis nigricans.

${ }^{\mathrm{b}} \mathrm{lg}$ : immunoglobulin-like loop.

'TK: tyrosine kinase.

TM : transmembrane. 
number of fused sutures or type of synostosis. Patients harbouring the novel FGFR2 mutations (Y308C, I288N and Y340S) had a typical form of CS. The uncommon L357S mutation was detected in a familial case and segregated with the disease. The proband was a 12-year-old girl with pansynostosis and chronic tonsillar herniation. Her older brother who had sagittal and bicoronal synostosis had never required surgery because facial anomalies were mild. Mutations in the tyrosine kinase domains were associated with hydrocephaly in one of the two patients carrying the $\mathrm{N} 549 \mathrm{H}$ substitution and slight developmental delay in the patient with the K641R mutation.

\section{JW patients}

An heterozygous dinucleotide deletion (AC) at position 958-959 was detected in exon 10 of FGFR2 in a familial form of JW variant with tarsal/metatarsal coalescence in the absence of hand anomalies (Figure 1a-d). This frameshift mutation that occurred in a mother and her son, was predicted to induce translation of four illegitimate amino acids, immediately followed by a premature termination codon (TGA) at position 324 of the receptor (Figure $2 b$ ). To determine if the 2 bp deletion affected stability of the IIIc transcript, oligonucleotides located in exon 8 and 10 were chosen to selectively amplify the IIIc transcript by RT-PCR.
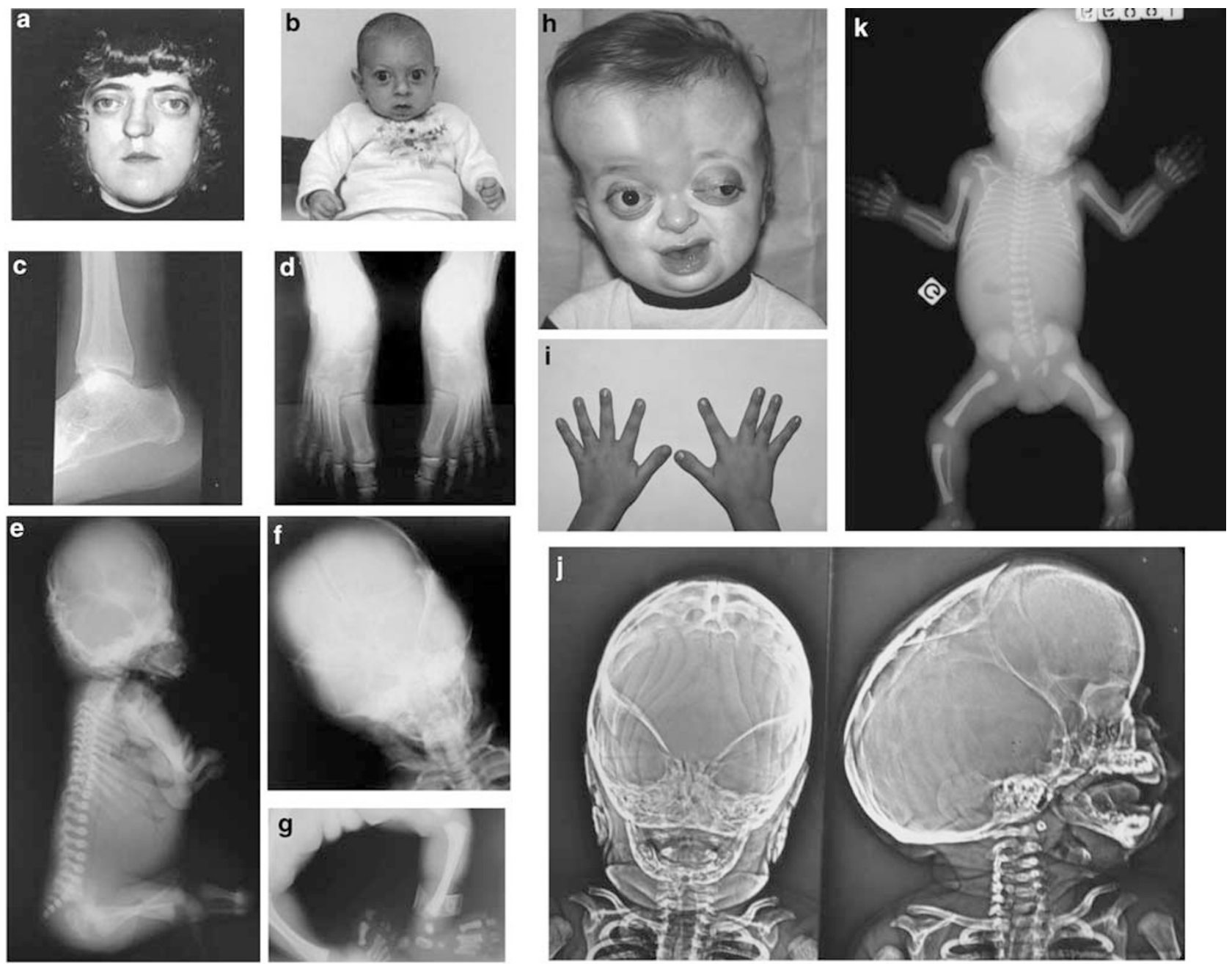

Figure 1 Clinical and radiological features of patients with JW phenotype and PS. (a, b) Presence of brachycephaly, midface hypoplasia, facial dysmorphism and proptosis in a mother and her son with JW phenotype. (c, d) Note partial tarsal fusion and extra delta-shaped small bone between the first and the second phalanx of the left hallux in the boy (at 15 years) and complete tarsal fusion in the mother. (e) Lateral and frontal radiographs of a 25-week-old foetus with PS carrying the Y340C FGFR2 mutation showing humero-radio-ulnar synostosis and sacral anomalies. (f) Cloverleaf skull. (g) Abnormal shape of the first phalanxes of big toes. (h) Severe proptosis, marked brachycephaly with underossified cranial vault, (i) mild syndactyly and flat thumbs in a patient with PS carrying a K641R FGFR2 mutation. (j) Facial and lateral radiographs of a Pfeiffer patient carrying a C342R FGFR2 mutation, showing severe multisynostoses of sagittal and coronal sutures responsible for severe scaphocephaly. (k) Radiograph of a 29-week-old foetus with PS carrying the W290C FGFR2 mutation showing brachycephaly with cloverleaf skull, bilateral humero-radio-ulnar synostosis and large and deviated thumbs. 
c

a

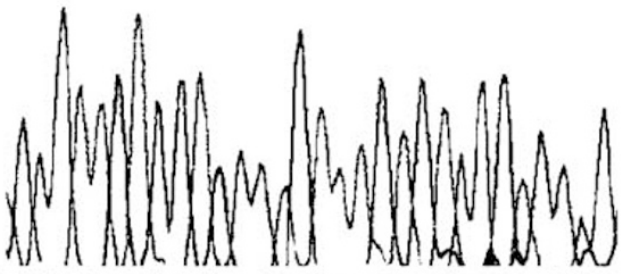

normal $[$ Val Asn, Thr, Thr Asp Lys, Giu, lle Glu, Val

allele GTTAACACCACGGACAAAGAGATTGAGGTT

mutated GTTAACACCGGACAAAGAGAT TGA

allete

b

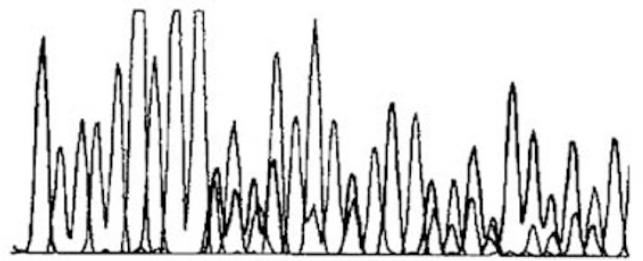

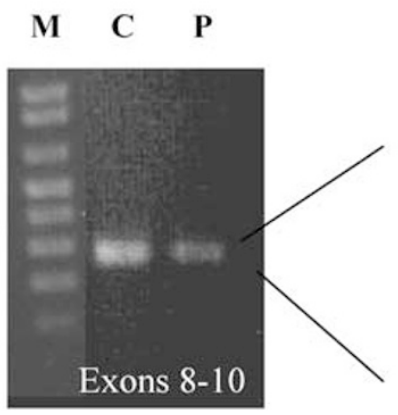

d
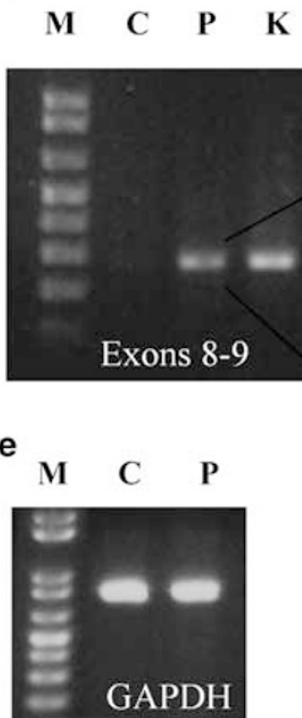

IIIc isoform

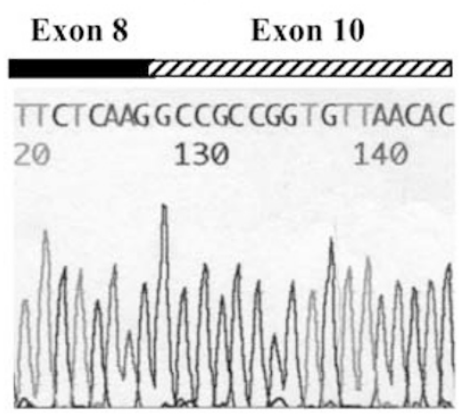

IIIb isoform

\section{Exon $8 \quad$ Exon 9}

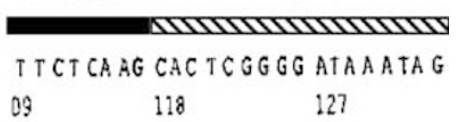

$09 \quad 118 \quad 127$

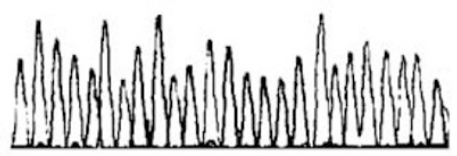

Figure 2 Sequencing of genomic DNA and RNA transcripts from the JW patient. (a) Direct sequencing of a normal allele (b) sequence of patient genomic DNA showing a heterozygous frameshift deletion (delAC) in exon 10 of FGFR2. (c) Agarose gel analysis and sequence of the RT-PCR product derived from control $(C)$ and patient $(P)$ fibroblasts by using a specific reverse primer for exon 10. A reduced amount of the patient's IIllc transcript is visible. The normal sequence of exon 10 indicates that the mutant allele lacking the 2 bp (AC) is not detectable at the cDNA level. (d) Agarose gel analysis and sequence of the RT-PCR product derived from control (C) and patient (P) fibroblasts and control keratinocytes (K) by using a reverse primer specific for exon 9. The sequence of the patient PCR product shows the expected exons 8-9 junction of the IIIb isoform. (e) RT-PCR of GAPDH used as an internal control. $M=$ molecular weight marker ( $1 \mathrm{~kb}+$ from In Vitrogen).

A 286-bp fragment of the expected size was visualized with RNA extracted from fibroblasts of the affected mother and an age-matched control, however, the intensity of the patient's band was markedly reduced. Sequencing of the patient RT-PCR product revealed only the normal sequence suggesting instability and degradation of the mutant IIIc transcript (Figure 2c). We then tested whether the mutation induced ectopic expression of the IIIb transcript. For this purpose, the same forward oligonucleotide in exon 8 was combined with a reverse oligonucleotide in exon 9. Control keratinocytes and patient's fibroblasts gave rise to a $281 \mathrm{bp}$ fragment, which upon sequencing proved to correspond to the IIIb trancript, while control fibroblasts gave almost no signal (Figure 2d). Similar results were obtained with a second set of primers located in exon 9 (not shown) indicating that illegitimate expression of the IIIb isoform in cells of mesenchymal origin had occurred.

\section{Pfeiffer patients}

FGFR mutations were detected in 19/20 (95\%) Pfeiffer samples. Three patients carried the recurrent P252R FGFR1 substitution that gave rise to a relatively mild phenotype (Table 3). In all, 16 patients including two familial forms and 14 sporadic cases harboured a FGFR2 mutation; two of them have been reported earlier. ${ }^{5}$ Although variable, the phenotype was more severe (Figure 1j) than in patients carrying FGFR1 mutations as attested by marked facial deformities, common hydrocephaly (in 10/16 patients), mental retardation and premature death (Table 3 ). In exon 8, the W290C FGFR2 mutation was identified in three 
Table 3 Clinical features of Pfeiffer patients carrying FGFR2 or FGFR1 mutations

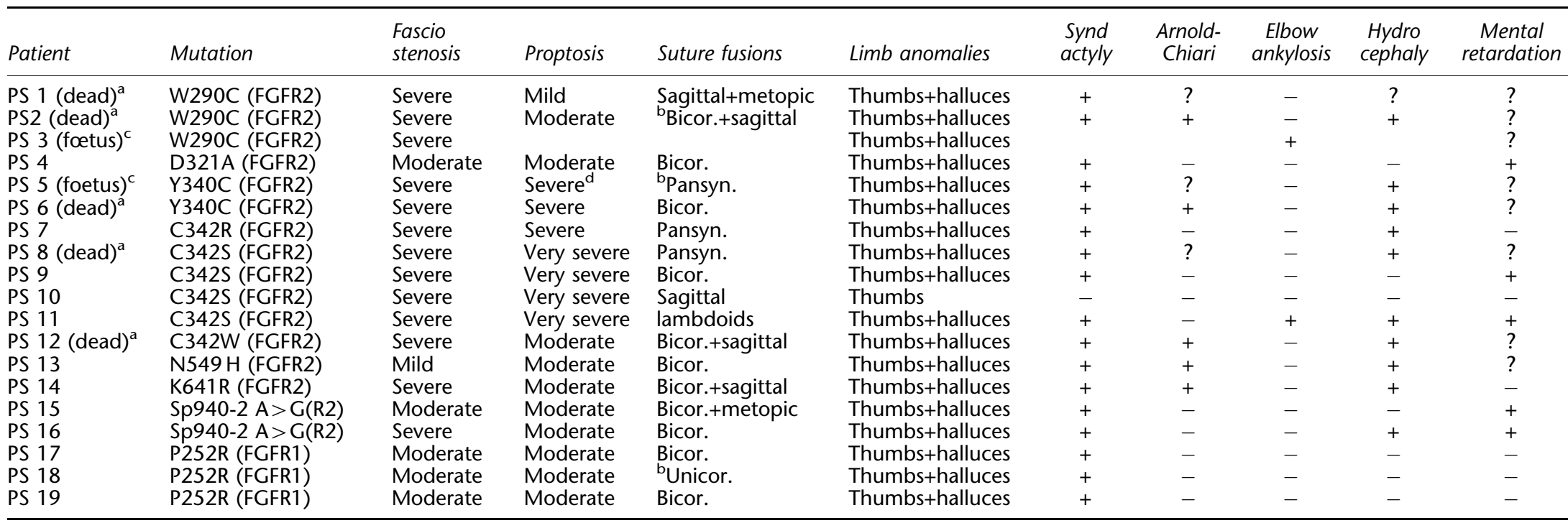

aPremature death due to respiratory distress.

${ }^{b}$ Bicor. = bicoronal, fusion of two coronal sutures (brachycephaly); Unicor. = unicoronal, fusion of one coronal suture (plagiocephaly); Pansyn. = pansynostosis, fusion of all sutures. cPregnancy was interrupted at 25 weeks after ultrasound examination and detection of multiple malformations.

${ }^{d}$ Cloverleaf skull. 
cases, two of them died prematurely and the third was prenatally diagnosed by ultrasound examination at 29 weeks of gestation. Radiological and clinical examination after termination of pregnancy confirmed the diagnosis of Pfeiffer type II with very severe proptosis, cloverleaf skull and humero-radio-ulnar synostosis (Figure 1k). In exon 10, we found nine mutations of five different types and at the intron-exon 10 junction, the common splice mutation $(940-2 \mathrm{~A} \rightarrow \mathrm{G})$ that disrupts an acceptor site, was detected. Both patients harbouring the Y340C mutation had a severe phenotype consistent with the diagnosis of Pfeiffer type II. One case died in the early childhood of respiratory distress while the second case was prenatally diagnosed and pregnancy was terminated at 25 weeks of gestation. Radiological examination showed cloverleaf skull, elbow ankylosis with bilateral humero-radio-ulnar synostosis, broad and deviated big toes and vertebral anomalies including sacrococcygeal eversion (Figure $1 \mathrm{e}-\mathrm{g}$ ).

Mutations in exons 14 and 16 encoding the tyrosine kinase domains TK1 and TK2 were identified in two sporadic cases. Mutation in the TK2 domain was associated with a more severe phenotype (Figure $1 \mathrm{~h}, \mathrm{i}$ ) than in the TK1.

\section{Discussion}

We have carried out a molecular study of three FGFR genes in a large series of 131 cases with a clinical diagnosis of AS, CS, PS and JW variant. On the basis of previous studies ${ }^{15,20}$ and our own experience, the mutation detection rate appears to depend largely on the accuracy of the original diagnosis and the sensitivity of the detection method. In our study, all patients were examined by the same physicians and complete clinical and radiological data were recorded, so that the criteria for phenotypic classification were highly homogeneous allowing recognition of the Crouzonoid facies.

Based on our clinical diagnosis and using direct sequencing, we identified FGFR2 mutations in 95\% (57/60) of our Crouzon patients. This situation is similar to two previous studies $^{15,21}$ in which mutations were detected in 18/20 and 25/28 Crouzon patients, respectively. In PS, we found $95 \%$ of mutations in our cohort of 20 patients. A similar percentage was obtained in the Oxford series. ${ }^{15}$ In contrast, Cornejo-Roldan et $a l^{20}$ studying a total of 78 unrelated Pfeiffer patients identified FGFR mutations in only 40 cases (51\%). The difference between the three studies could rely on the stringency of clinical diagnosis. Only patients showing hands and feet anomalies (deviation and broadening of thumbs and big toes) were included in our PS group (Table 3). In the Cornejo-Roldan's cohort, the criteria for inclusion in the Pfeiffer group were presumably too broad. Hence, the presence of mild radiological findings such as shortened middle phalanges might be insufficient to make a diagnosis of PS.

In keeping with previous reports, ${ }^{22,23}$ analysis of aminoacid substitutions in our series and comparison with data from other groups indicated that trends in genotypephenotype correlation seemed to emerge. As shown in Table 4, amino-acid substitutions at residues 267, 278, 289, 338, 347 and 354 mostly accounted for CS (59/65 cases, $90 \%$ ). Common substitutions affecting amino acids W290, Y340 and C342 were of particular interest since the phenotype appeared to rely in most cases on the newly

Table 4 Genotype-phenotype analyses in CS, JW and PS patients carrying FGFR2 mutations

\begin{tabular}{|c|c|c|c|c|c|}
\hline FGFR2 mutation & Crouzon & $\begin{array}{c}\text { Number of cases } \\
\text { Jackson-Weiss }\end{array}$ & Pfeiffer & Predominant phenotype & $N b$ of cases/Total $n b^{b}$ \\
\hline S267P/F & 6 & - & 1 & CS & $6 / 7$ \\
\hline $\mathrm{C} 278 \mathrm{~F}$ & 20 & 1 & 3 & CS & $20 / 24$ \\
\hline Q289P & 8 & 1 & - & CS & $8 / 9$ \\
\hline W290G & 3 & - & - & CS & $3 / 3$ \\
\hline W290R & 5 & - & - & CS & $5 / 5$ \\
\hline W290C & - & - & 9 & PS & $9 / 9$ \\
\hline G338R & 8 & - & - & CS & $8 / 8$ \\
\hline Y340H & 8 & - & - & CS & $8 / 8$ \\
\hline Y340C & - & - & 5 & PS & $5 / 5$ \\
\hline C342S & 6 & 2 & 12 & PS & $12 / 20$ \\
\hline C342F & 4 & - & - & CS & $4 / 4$ \\
\hline C342Y & 43 & - & 3 & CS & $43 / 46$ \\
\hline C342R & 2 & 1 & 22 & PS & $22 / 25$ \\
\hline S347C & 8 & - & - & CS & $8 / 8$ \\
\hline S354C/F & 9 & - & - & CS & $9 / 9$ \\
\hline A344A & 10 & - & - & CS & $10 / 10$ \\
\hline $940-2 A>G$ & - & - & 15 & PS & $15 / 15$ \\
\hline
\end{tabular}

${ }^{a}$ Numbers were obtained by combining our findings with previous data from several groups. ${ }^{3,4,6,10,13-16,20,21,24-26,28,34,35}$ Only mutations corresponding to a significant number of cases were included in the table.

${ }^{\mathrm{b}}$ Ratios represent the number of cases exhibiting the predominant phenotype over the total number of cases reported to carry the mutation. 
created residue. Hence, conversion of tryptophan 290 into arginine or glycine resulted in a Crouzon phenotype in 8/8 cases while conversion into cysteine produced a severe form of PS (often leading to premature death) both in our series and in six previous cases. ${ }^{13,15,24-26}$ An additional patient presented a severe nonclassifiable craniosynostosis syndrome with limb and joint anomalies and mental retardation. ${ }^{27}$ Tyrosine 340 when converted into histidine gave rise to CS in $8 / 8$ patients (Table 4). By contrast conversion into cysteine reproducibly led to very severe forms of $\mathrm{PS}^{15,20,28}$ with cloverleaf skull, severe ocular proptosis, hydrocephalus, and early demise in some cases (Table 3). Mutations eliminating cysteine 342 were also noticeable with respect to phenotype. Conversion into phenylalanine and tyrosine (two amino-acids with aromatic lateral chains) mainly produced CS in $47 / 51$ patients whereas conversion into arginine preferentially caused PS (22/25 cases). A similar situation exists in FGFR3-related skeletal dysplasias. Substitutions of lysine 650 by glutamic acid or methionine residues give rise to thanatophoric dysplasia or SADDAN whereas replacement of lysine by glutamine, asparagine or threonine generates hypochondroplasia, a much milder condition. ${ }^{29,30}$ Differences in the extent of receptor activation due to conformational changes of the receptor three-dimensional structure induced by amino-acids of variable charge, size or hydrophobicity could explain this result. Disruption of the acceptor splice site of exon $10(940-2 \mathrm{~A}>\mathrm{G})$ and creation of a cryptic splice donor site (A344A) resulted in distinct phenotypes, the former being associated with PS and the latter with CS (Table 4).

These observations strongly suggest that PS is accounted for by a limited number of mutations and that at least three specific amino-acid changes W290C, Y340C and C342R are associated with severe forms of PS characterized by multiple malformations including defective neurological functions and respiratory distress secondary to tracheal cartilage sleeves ${ }^{23,25}$ (Table 3). Interestingly, another amino-acid substitution creating an unpaired cysteine residue, namely S351C, also results in severe forms of PS in most reported cases. ${ }^{31-33}$ Although confusion might exist between severe PS and the so-called 'Antley-Bixler-like' phenotype, ${ }^{34,35}$ we consider that the heterozygous S351C FGFR2 mutation is a hallmark of severe forms of PS, given that typical forms of Antley-Bixler syndrome are caused by recessive mutations in the $\mathrm{P} 450$ oxido-reductase (POR) gene. $^{36}$

Altogether, these data indicate that the presence of craniosynostosis and multiple anomalies evocative of severe PS (type II or III) should be confirmed at the molecular level by analysing recurrent FGFR2 mutations (W290C, Y340C, C342R and S351C) first. Owing to the poor clinical outcome of patients carrying these mutations, molecular diagnosis at birth or soon after should be associated with intensive care in specialized Unit for treatment of pulmonary problems. Only when recurrent mutations are not found, should sequencing of additional exons be undertaken.

So far, FGFR2 mutations causing craniosynostoses proved to be gain-of-function mutations resulting in either ligandindependent activation of the receptor ${ }^{37}$ or enhancing the affinity of ligand binding ${ }^{38}$ or producing illegitimate ligand binding and signalling. ${ }^{39,40}$ Consequently, the identification of a 2 bp deletion in a familial form of JW variant causing the appearance of a putative premature termination codon was somewhat unexpected. RT-PCR analysis of FGFR2 transcripts revealed the instability of the mutant IIIc isoform leading to illegitimate expression of the alternative splice product IIIb in fibroblasts. This complex process may involved increased binding of ribonuclear proteins (RNP) to regulatory intronic sequences controlling exon 9 (IIIb) splicing. ${ }^{41,42}$ Interestingly, it has been previously demonstrated that Alu-element insertion upstream or within exon 10 (IIIc) of FGFR2-induced ectopic expression of the IIIb isoform in one Apert patient. ${ }^{43}$ The same effects were observed in four patients with PS carrying the splice mutation $940-2 \mathrm{~A}>\mathrm{G} / \mathrm{T}^{43,44}$ Likewise, hemizygous deletion of exon IIIc in a murine model induced ectopic expression of the IIIb (KGFR) isoform. ${ }^{45}$ By contrast, in another mouse model, insertion of a frameshift mutation in exon IIIc did not influence the expression of the IIIb isoform at early stages of development. ${ }^{46}$ The discrepancy between this mouse model and the human situation might be related to differences in the developmental stages (human adult versus mouse embryo) and/or homozygosity of mutant animals versus heterozygosity of human mutations. Since FGFR2 mutations causing Apert syndrome and some cases of PS alter FGF binding specificity or result in ectopic FGFR2 IIIb expression, ${ }^{38,39,43}$ we speculate that in our case, inappropriate expression of the IIIb isoform in mesenchymal cells would allow binding and signalling through FGF10 or FGF7 ligands, leading to a gain-of-function mechanism. If we assume that limb anomalies in JW variants, PS and AS require threshold signalling to become detectable, it would mean that the level of expression of the IIIb isoform in mesenchymal cells of our patient was lower than in previously reported Pfeiffer patients, ${ }^{43,44}$ and that lower limbs are more sensitive to illegitimate activation than upper limbs.

\section{Acknowledgements}

We thank patients and their families for their participation in this study.

\section{References}

1 Hall CM: International nosology and classification of Constitutional disorders of bone, 2005, 7th International Skeletal Dysplasia Meeting Martigny, Switzerland.

2 Cohen Jr MM: Pfeiffer syndrome update, clinical subtype and guidelines for differential diagnosis. Am J Med Genet 1993; 45: 300-307. 
3 Reardon W, Winter RM, Rutland $\mathrm{P}$ et al: Mutations in the fibroblast growth factor receptor 2 gene cause Crouzon syndrome. Nat Genet 1994; 8: 98-102.

4 Jabs EW, Li X, Scott AF et al: Jackson-Weiss and Crouzon syndromes are allelic with mutations in fibroblast growth factor receptor 2. Nat Genet 1994; 8: 275-279.

5 Lajeunie E, Ma HW, Bonaventure J et al: FGFR2 mutations in Pfeiffer syndrome. Nat Genet 1995; 9: 108.

6 Rutland P, Pulleyn LJ, Reardon W et al: Identical mutations in the FGFR2 gene cause both Pfeiffer and Crouzon syndrome phenotypes. Nat Genet 1995; 9: 173-176.

7 Wilkie AOM, Slaney SF, Oldridge M et al: Apert syndrome results from localized mutations of FGFR2 and is allelic with Crouzon syndrome. Nat Genet 1995; 9: 165-172.

8 Przylepa KA, Paznekas W, Zhang M et al: Fibroblast Growth Factor Receptor 2 mutations in Beare-Stevenson cutis gyrata syndrome. Nat Genet 1996; 13: 492-494.

9 Muenke M, Schell U, Hehr A et al: A common mutation in the fibroblast growth factor receptor 1 gene in Pfeiffer syndrome. Nat Genet 1994; 8: 269-274.

10 Schell U, Hehr A, Feldman GJ et al: Mutations in FGFR1 and FGFR2 cause familial and sporadic Pfeiffer syndrome. Hum Mol Genet 1995; 4: 323-328.

11 Moloney DM, Slaney SF, Oldridge $\mathrm{M}$ et al: Exclusive paternal origin of new mutations in Apert syndrome. Nat Genet 1996; 13: $48-53$

12 Glaser RL, Jiang W, Boyadjiev SA et al: Paternal origin of FGFR2 mutations in sporadic cases of Crouzon Syndrome and Pfeiffer syndrome. Am J Hum Genet 2000; 66: 768-777.

13 Tartaglia M, Valeri S, Velardi F et al: Trp290cyst mutation in exon IIIa of the fibroblast growth factor receptor 2 (FGFR2) gene is associated with Pfeiffer syndrome. Hum Genet 1997; 99: 602-606.

14 Passos-Bueno MR, Wilcox WR, Jabs EW et al: Clinical spectrum of Fibroblast Growth Factor Receptor mutations. Hum Mutat 1999; 14: $115-125$.

15 Kan S, Elanko N, Johnson D et al: Genomic screening of fibroblast growth factor receptor 2 reveals a wide spectrum of mutations in patients with syndromic craniosynostosis. Am J Hum Genet 2002; 70: $472-486$.

16 Meyers GA, Orlow SJ, Munro IR, Przylepa KA, Jabs EW: Fibroblast Growth Factor Receptor 3 (FGFR3) transmembrane mutation in Crouzon syndrome with acanthosis nigricans. Nat Genet 1995; 11: $462-464$.

17 Ingersoll RG, Paznekas WA, Tran AK et al: Fibroblast growth factor 2 (FGFR2): genomic sequence and variations. Cytogenet Cell Genet 2001; 94: 121-126.

18 Lajeunie E, Cameron R, El Ghouzzi V et al: Clinical variability in patients with Apert's syndrome. J Neurosurg 1999; 90: 443-447.

19 Ma HW, Lajeunie E, Le Merrer M et al: No evidence of genetic heterogeneity in Crouzon craniofacial dysostosis. Hum Genet 1995; 96: 731-735.

20 Cornejo-Roldan LR, Roessler E, Muenke M: Analysis of the mutational spectrum of the FGFR2 gene in Pfeiffer syndrome. Hum Genet 1999; 104: 425-431.

21 Kress W, Collmann H, Büsse M, Halliger-Keller B, Mueller CR: Clustering of FGFR2 gene mutations in patients with Pfeiffer and Crouzon syndromes (FGFR2-associated craniosynostoses). Cytogenet Cell Genet 2000; 91: 134-137.

22 Wilkie AOM, Patey SJ, Kan S-H et al: FGFs, their receptors and human limb malformations: clinical and molecular correlations. Am I Med Genet 2002; 112: 266-278.

23 Cohen Jr MM: FGFs/FGFRs and associated disorders; in Epstein CJ, Erickson RP, Wynshaw-Boris A (eds): Inborn errors of development. New York: Oxford University press, 2004, pp 380-400.

24 Schaefer F, Anderson C, Can B, Say B: Novel mutation in the FGFR2 gene at the same codon as the Crouzon syndrome mutations in a severe Pfeiffer syndrome type 2 case. Am J Med Genet 1998; 75: 252-255.

25 Zackai EH, McDonald-McGinn DM, Stolle C, Huff DS: Craniosynostosis with tracheal sleeve: a patient with Pfeiffer syndrome, tracheal sleeve and additional malformations in whom an FGFR2 mutation was found. Clin Dysmorphol 2003; 12: 209.

26 Nazarro A, Della Monica M, Lonardo F et al: Prenatal ultrasound diagnosis of a case of Pfeiffer syndrome without cloverleaf skull and review of the literature. Prenat Diagn 2004; 24: 918-922.

27 Shotelersuk V, Ittiwut C, Srivuthana S et al: Distinct craniofacialskeletal-dermatological dysplasia in a patient with W290C mutation in FGFR2. Am J Med Genet 2002; 113: 4-8.

28 Blaumeiser B, Loquet P, Wuyts W, Nothen MM: Prenatal diagnosis of Pfeiffer syndrome type II. Prenat Diagn 2004; 24: 644-646.

29 Bellus GA, Gaudenz K, Zakai EH et al: Identical mutations in three different fibroblast growth factor receptor genes in autosomal dominant craniosynostosis syndromes. Nat Genet 1996; 14: $174-175$.

30 Bellus GA, Spector EB, Speiser PW et al: Distinct missense mutations of the FGFR3 Lys 650 codon modulate receptor kinase activation and the severity of the skeletal dysplasia phenotype. Am J Hum Genet 2000; 67: 1411-1421.

31 Gripp KW, Stolle CA, McDonald-McGinn DM et al: Phenotype of the fibroblast growth factor receptor 2 Ser 351Cys mutation: Pfeiffer syndrome type III. Am J Med Genet 1998; 78: 356-360.

32 Sweeney E, Ellis I, May P: Sacral appendage associated with a mutation in FGFR2. Clin Dysmorphol 2002; 11: 221-222.

33 Gonzales M, Heuertz S, Martinovic J et al: Vertebral anomalies and cartilagineous tracheal sleeve in three patients with Pfeiffer syndrome carrying the S351C FGFR2 mutation. Clin Genet 2005; 68: $179-181$.

34 Reardon W, Smith A, Honour JW et al: Evidence for digenic inheritance in some cases of Antley-Bixler syndrome. J Med Genet 2000; 37: 26-32.

35 Tsai F-J, Wu J-Y, Yang C-F, Tsai C-H: Further evidence that fibroblast growth factor receptor 2 mutations cause Antley-Bixler syndrome. Acta Paediatr 2001; 90: 595-597.

36 Fluck CE, Tajima T, Pandey AY et al: Mutant P450 oxidoreductase causes disordered steroidogenesis with and without Antley-Bixler syndrome. Nat Genet 2004; 36: 228-230.

37 Robertson SC, Tynan JA, Donoghue DJ: RTK mutations and human syndromes: when good receptors turn bad. Trends Genet 2000; 16: $265-271$

38 Anderson J, Burns HD, Enriquez-Harris P et al: Apert syndrome mutations in fibroblast growth factor receptor 2 exhibit increased affinity for FGF ligand. Hum Mol Genet 1998; 7: 1475-1483.

39 Yu K, Herr AB, Waksman G, Ornitz DM: Loss of fibroblast growth factor receptor 2 ligand-binding specificity in Apert syndrome. Proc Natl Acad Sci USA 2000; 97: 14536-14541.

40 Ibrahimi OA, Zhang F, Eliseenkova AV et al: Biochemical analysis of pathogenic ligand-dependent FGFR2 mutations suggests distinct pathophysiological mechanisms for craniofacial and limb abnormalities. Hum Mol Genet 2004; 13: 2313-2324.

41 Del Gatto F, Plet A, Gesnel A et al: Multiple interdependent sequence elements control splicing of a Fibroblast Growth factor Receptor 2 alternative exon. Mol Cell Biol 1997; 17: 5106-5116.

42 Carstens RP, Wagner EJ, Garcia-Blanco MA: An intronic splicing silencer causes skipping of the IIIb exon of fibroblast growth factor receptor 2 through involvement of polypyrimidine tract binding protein. Mol Cell Biol 2000; 20: 7388-7400.

43 Oldridge M, Zackai EH, McDonald-McGinn DM et al: De novo Aluelement insertions in FGFR2 identify a distinct pathological basis for Apert syndrome. Am J Hum Genet 1999; 64: 446-461.

44 Tsukuno M, Suzuki H, Eto T: Pfeiffer syndrome caused by haploinsufficient mutation of FGFR2. J Craniofac Genet Dev Biol 1999; 19: 183-188.

45 Hajihosseini MK, Wilson D, de Moerlooze L, Dickson C: A splicing switch and gain-of-function mutation in FGFR2-IIIc hemizygotes causes Apert/Pfeiffer-syndrome-like phenotypes. Proc Natl Acad Sci USA 2001; 2798: 3855-3860.

46 Eswarakumar VP, Monsonego-Ornan E, Pines M et al: The IIIc alternative of FGFR2 is a positive regulator of bone formation. Development 2002; 129: 3783-3793. 